\title{
Control of enzymatic reactions via quadratic immersion
}

Francesco Carravetta

\begin{abstract}
One of the aims of Synthetic Biology is to regulate metabolic products for specific biochemical reaction networks. These networks are usually designed as elementary modules to be interconnected each other and efforts are spent to minimize undesired coupling effects arising from the mutual interaction. Within this framework, this note considers a basic enzymatic reaction scheme as an elementary input/output module, with a related specific control problem addressed according to a quadratic immersion, a recently developed control methodology. The quadratic immersion embeds the system equations that model the enzymatic reaction network into an extended, finitedimensional state-space, according to which the control problem may be restated in a simplified version, whose solution guarantees the output asymptotic convergence to a desired value according to a smooth trajectory.
\end{abstract}

Index Terms-Nonlinear Systems, Enzymatic Reactions, Synthetic Biology

\section{INTRODUCTION}

Synthetic Biology is a challenging and quite recent research field, aiming at engineering artificial biological systems, potentially useful for biotechnology industry, human health and environment (see [4], [14], [12] and references therein). To this end, modularity is considered an unavoidable frame, and efforts are devoted to minimize undesired coupling effects arising from the interaction of mutually interconnected modules [13], [11]. Indeed, recent results showed how synthetic feedback control schemes could be implemented by suitably designing chemical reaction networks to work as building blocks of a larger interconnected embedded system [3], [15], [24], [10].

In this work a control problem is addressed for a basic enzymatic reaction scheme, which can be exploited as a standard toy-model for any kind of enzymatic reaction. Enzymes are known to play a relevant role in many and diverse cellular activities (like metabolism, signal transduction and cell regulation), and their malfunction may lead to the raise of serious diseases [8], [9]. Further interest has recently gained from the practical applications of enzymes as specific catalysts in drug development, food processing and biofuel production [20]. These facts explains the interest in predictive mathematical models associated to such elementary chemical reaction network, resumed in Fig. 1: a substrate $S$ that is transformed

F. Carravetta and P. Palumbo are with the Istituto di Analisi dei Sistemi e Informatica "A. Ruberti", National Research Council (IASI-CNR), Via dei Taurini 19, 00185 Rome, Italy. E-Mail Addresses: \{francesco.carravetta, pasquale.palumbo\}@iasi.cnr.it

\author{
Pasquale Palumbo
}

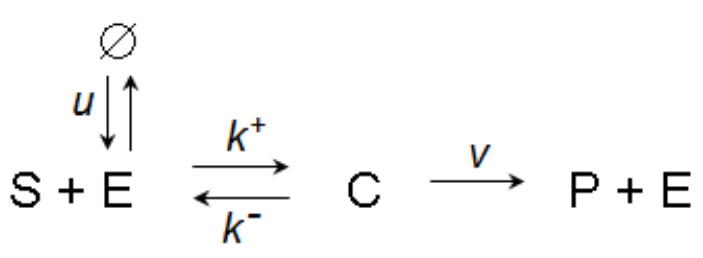

Fig. 1. Basic scheme of enzymatic reactions.

into a product $P$ by means of the catalytic action of an enzyme $E$ that first forms a complex $C$ according to a reversible binding/unbinding reaction and, then, releases the product according to a not-reversible reaction. This network may be seen as an input/output system, with the output provided by the accumulation of the final product $P$, and the control action exerted by varying the enzyme concentration. The goal of the control law is to regulate the concentration of the final product $P$, possibly according to a chosen smooth trajectory. In this work we neglect random fluctuations, thus assuming to have a number of molecules of each species large enough to rely on Ordinary Differential Equation (ODE) models describing average concentrations that vary continuously [25].

The approach followed is based on the quadratic immersion [7], a procedure that allows to embed a nonlinear system into a larger, finite-dimensional state space. According to nonrestrictive conditions, the quadratic immersion may be applied to a large class of nonlinear ODE systems, to which the one describing the enzymatic reaction scheme belongs. Such embedding allows to restate the original nonlinear dynamic equations into a homogenous quadratic form. When applied to the system under investigation, we find out that the control input enters the quadratic immersion in a way that allows to decouple the original control problem into a cascade of elementary steps. The main result provides a closed-loop control that ensures the final product asymptotic convergence to a desired value according to a smooth trajectory.

\section{Problem Setting}

\section{A. Mathematical model of a basic enzymatic reaction scheme}

Consider the chemical reaction network reported in Fig.1, representing a classical enzymatic reaction scheme. According to the deterministic approach, the state of the system is provided by the species concentrations, here denoted by $S$, $C, P$ and $E$. The ODE dynamics is written below according 
to standard mass action law.

$$
\begin{aligned}
& \dot{S}(t)=-k^{+} S(t) E(t)+k^{-} C(t) \\
& \dot{C}(t)=k^{+} S(t) E(t)-\left(k^{-}+v\right) C(t) \\
& \dot{P}(t)=v C(t) \\
& \dot{E}(t)=-k^{+} S(t) E(t)+\left(k^{-}+v\right) C(t)+u(t)
\end{aligned}
$$

The control action is exerted on the enzyme $E$, with the control input $u$ denoting the external flux control on $E$. Here we assume that $u$ may be both positive or negative, i.e. it may work as an inward/outward exchange flux. In other words we allow to add or to spill out the enzyme at a desired rate. One way to diminish one species concentration is by means of molecular sequestration (see, e.g. [3] where it has been exploited to design molecular controllers that perform integral action). In any case, herein we do not address how to effectively achieve such control; instead we just allow the control input to assume positive or negative values.

Notice that the presence of external fluxes modeled by the control input $u$ makes the chemical reaction scheme an open system, since the overall enzyme concentration is not conserved. On the other hand, the overall substrate concentration (provided by the sum of the unbound substrate $S$, the complex $C$ and the final product $P$ ) is conserved, as it straightforwardly comes from the sum of such three time-derivatives, identically equal to zero.

\section{B. The regulation problem}

The regulation problem consists in designing the control law $u(t)$ in order to track a desired stationary value for $P(t)$, possibly according to a smooth trajectory. Before to introduce our control proposal, we just briefly comment on existing methods of nonlinear control that could in principle be used to solve the regulation problem for a system of the type (1). System (1) is a quadratic differential equation for which most of the existing control methods cannot yield the solution of the regulation problem in a systematic way. For instance, the optimal control approach would give place to the HamiltonJacobi-Bellmann (HJB) equations, with no analytical solution unless trivial cases. We refer readers to [1], [2] and [21], for a theoretical account as well as for methods providing approximate solutions of the HJB equation. Lyapunov based methods require the determination (non trivial, as well, in most cases) of a Lyapunov function on the basis of the system equations; related papers, among others of a wide literature on this subject, include also ISS (Input-to-State Stability) based methods, such as [23], [18]. Also, the application of the feedback linearization [17] would require the not easy determination of a full relative degree function for system (1): indeed none of the state variables in (1) can be referred to as such an output function with full relative degree, and the search for it would mean to look for a solution (in closed form) of the partial differential equations describing the binding between such a function and the diffeomorphism providing the linearized system coordinates.

The solution that we propose here, is based on the exact quadratization procedure developed in [7], (see also the former papers [5], [6] for case studies and examples of applications), where it is proved that a very large class of nonlinear systems, namely those which are analytic and in Integral Closed Form (ICF systems), can be re-written as a second-order homogenous ODE by systems immersion, a procedure that allows to embed the original nonlinear system into a larger, finitedimensional state space. The class of systems to which exact quadratization can be applied is very large. Indeed, the ICF systems include all systems' functions expressed by a finite composition of (i) elementary algebraic operations (sums, products, powers of real exponents), (ii) common transcendental functions (sin, cos, exponential, logarithm, etc.) or (iii) integrals of the above functions, with bounds expressed by any of them. One of the advantages of the quadratic immersion is that the dynamics related to each entry of the augmentedstate, may depend of (possibly) all control inputs. This sort of input 'shuffle' makes the quadratizing transformation of interest even in control problems for quadratic systems, like the enzymatic reaction model at issue. Moreover, the quadratization procedure benefits of two further nice properties. First, it is easy to implement, because all the transformations providing the new state variables are predetermined, i.e. they are readily obtained in a systematic way from the equations of the original system. Finally, the embedding transformation does not modify the original system dynamics: indeed, the new state vector is a larger one which simply includes the original state as a sub-vector, and thus the control designer is not concerned with inverting the transformation in order to get the system back in the original coordinates.

\section{Quadratic Immersion}

We briefly summarize the concept of quadratic immersion, and refer the reader to [7] for all the details.

A systems immersion (resp. a dense immersion) (cf. [7][19]-[16]) from a system, $\mathcal{S}_{1}$ in $\mathbb{R}^{n}$, into another system $\mathcal{S}_{2}$ living in $\mathbb{R}^{m}$ (with $m \geq n$ ) is a smooth map from the domain (resp. the domain with possibly the exception of a zero-measure set) of $\mathcal{S}_{1}$ onto a smooth manifold, say $\mathcal{M}$, included in the domain of $\mathcal{S}_{2}$, such that any trajectory of $\mathcal{S}_{2}$ starting from $\mathcal{M}$ includes a trajectory of $\mathcal{S}_{1}$, and all the trajectories of $\mathcal{S}_{1}$ can be generated by trajectories of $\mathcal{S}_{2}$ starting from $\mathcal{M}$. A quadratic immersion is a dense immersion into a quadratic system.

Now, let us consider a $\sigma \pi$-algebraic system, ( $\sigma \pi$-system for short) that is a system of the following form (written component wise for $i=1, \ldots, n)$ :

$$
\begin{aligned}
& \dot{x}_{i}=\sum_{l=1}^{\nu_{i}} w_{i, l} X_{i, l}, \\
& X_{i, l}=\prod_{j=1}^{n} x_{j}^{p_{i, j}^{(l)}}
\end{aligned}
$$

where $\nu_{i}$ are non negative integers $\left(\nu_{i}=0\right.$ meaning that the $i$-th state equation is $\left.\dot{x}_{i}=0\right)$, the $w_{i, l}$ are (time-varying in 
general) parameters, or controls ${ }^{1}, X_{i, l}$ are said monomials, defined in (3), and the exponents $p_{i, j}^{(l)}$ are real numbers. The basic results proved in [7] is the following: the (scalar) variables

$$
Z_{i}^{(l)}=\frac{X_{i, l}}{x_{i}}
$$

satisfy the following system of ordinary differential equations:

$$
\dot{Z}_{i}^{(l)}=\left(\sum_{j=1}^{n} p_{i, j}^{(l)} Z_{j}^{T} w_{j}-Z_{i}^{T} w_{i}\right) Z_{i}^{(l)},
$$

where $w_{j}^{T}=\left[w_{j, 1}^{T}, \ldots, w_{j, \nu_{j}}^{T}\right], Z_{j}^{T}=\left[Z_{j}^{(1)^{T}} \ldots, Z_{j}^{\left(\nu_{j}\right)^{T}}\right]$, which constitute a new system, written componentwise for $i=1, \ldots, n$ and $l=1, \ldots, \nu_{i}$, of $d$ homogeneous quadratic differential equations where

$$
d=\sum_{i=1}^{n} \nu_{i}
$$

is the number of monomials in system (2). The quadratic system (5) is said the driver associated to the $\sigma \pi$-system (2). The name 'driver' comes from the fact that the general solution of a $\sigma \pi$ system can be written as a function of the solution of the associated driver. Indeed, looking at (2), (3), and (4) we have

$$
\dot{x}_{i}=\left(\sum_{l=1}^{\nu_{i}} Z_{i}^{(l)} w_{i, l}\right) x_{i}=\left(Z_{i}^{T} w_{i}\right) x_{i},
$$

whose solution is

$$
x_{i}=e^{\int_{o}^{t} Z_{i}^{T} w_{i}} x_{i}(0),
$$

and thus the quadratic system described by eqs. (5) can be thought of as 'driving' the 'final stage', i.e. another system given by eqs. (7), which is said the final system, and giving back the original state components. The map $x \mapsto Z$ has the same domain as the original $\sigma \pi$-system, unless possibly sets of type $\left\{x \in \mathbb{R}^{n}: x_{i} \neq 0\right\}$ which have a zero measure in $\mathbb{R}^{n}$. Moreover, if the driver (5) starts from $Z(\bar{x})$, it gives the original state $x$ through eq. (8). Thus the map $x \mapsto Z$ is a dense immersion into the quadratic system constituted by (5), (7), that is: a quadratic immersion. From (5) we see, as brought forward in section 2 , that all the drivers components depend of all the coefficients (and, hence, of all the controls), and (by (7)) the original state components depend each only of some of the driver components. What could make a driver component independent of some entry of the control $u$ is the exponent in a well determined monomial (see (3)) that could well be zero.

\footnotetext{
${ }^{1}$ Actually, they are either parameters or control-dependent coefficients, in that they might be non independent of each other. In general they are a linear combination of a set of independent controls. We have omitted here this detail for sake of simplicity: for the problem here at issue such a distinction does not apply since we have concern with a single scalar control $u$.
}

III. MAIN RESULTS

A. Applying the quadratic immersion to the enzymatic reaction model

According to the quadratic immersion, we define the driver state variables (4) as

$$
Z=\left[\begin{array}{c}
Z_{1}^{(1)} \\
Z_{1}^{(2)} \\
Z_{2}^{(1)} \\
Z_{3}^{(1)} \\
Z_{4}^{(1)} \\
Z_{4}^{(2)} \\
Z_{4}^{(3)}
\end{array}\right]=\left[\begin{array}{c}
E \\
C / S \\
E S / C \\
C / P \\
S \\
C / E \\
1 / E
\end{array}\right]
$$

where $Z_{2}^{(2)}=1$ has been clearly neglected. According to the formalism introduced by (2)-(4) we have:

$$
\nu_{1}=2, \quad \nu_{2}=2, \quad \nu_{3}=1, \quad \nu_{4}=3
$$

with

$$
\begin{array}{ll}
w_{1,1}=-k^{+}, & w_{1,2}=k^{-} \\
w_{2,1}=k^{+}, & w_{2,2}=-\left(k^{-}+v\right) \\
w_{3,1}=v & \\
w_{4,1}=-k^{+}, & w_{4,2}=k^{-}+v, \quad w_{4,3}=u(t)
\end{array}
$$

and

$$
\begin{array}{llrl}
p_{1,1}^{(1)}=1, & p_{1,4}^{(1)}=1, & p_{1,2}^{(2)}=1 \\
p_{2,1}^{(1)}=1, & p_{2,4}^{(1)}=1, & p_{2,2}^{(2)}=1 \\
p_{3,2}^{(1)}=1, & p_{4,1}^{(1)}=1, & p_{4,4}^{(1)}=1 \\
p_{4,2}^{(2)}=1, & p_{i, j}^{(l)}=0, & \text { elsewhere }
\end{array}
$$

from which the driver equations (5) are readily computed. More in details, from the final stage equation given in (7), the product dynamics can be written as:

$$
\dot{P}(t)=v Z_{3}^{(1)}(t) P(t),
$$

that may be thought of as a scalar system driven by the input $Z_{3}^{(1)}$, whose dynamics is given by (from (5)):

$\dot{Z}_{3}^{(1)}(t)=k^{+} Z_{3}^{(1)}(t) Z_{2}^{(1)}(t)-\left(k^{-}+v\right) Z_{3}^{(1)}(t)-v\left(Z_{3}^{(1)}\right)^{2}(t)$

Analogously, $Z_{3}^{(1)}$ may be thought of as a scalar system driven by the input $Z_{2}^{(1)}$ which, in turn, obeys the following dynamics (from (5)):

$$
\dot{Z}_{2}^{(1)}(t)=\varphi_{1}(Z(t))+\varphi_{2}(Z(t)) u(t)
$$

with

$$
\begin{aligned}
& \varphi_{1}(Z)=-k^{+} Z_{1}^{(1)} Z_{2}^{(1)}+k^{-} Z_{1}^{(2)} Z_{2}^{(1)}-k^{+}\left(Z_{2}^{(1)}\right)^{2} \\
& \quad+\left(k^{-}+v\right) Z_{2}^{(1)}-k^{+} Z_{2}^{(1)} Z_{4}^{(1)}+\left(k^{-}+v\right) Z_{2}^{(1)} Z_{4}^{(2)}(16) \\
& \varphi_{2}(Z)=Z_{2}^{(1)} Z_{4}^{(3)} .
\end{aligned}
$$

Thus, by means of (13), (14), and (15), we recognize a cascade of input/output scalar systems from the control input $u$ till the 


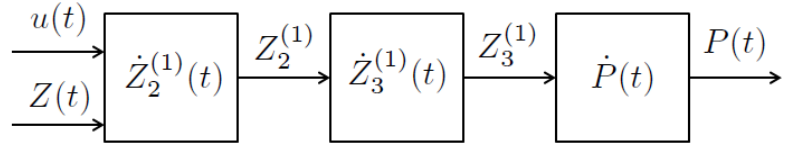

Fig. 2. Input/output scalar cascade provided by the quadratic immersion.

variable of interest $P: u$ drives $Z_{2}^{(1)}, Z_{2}^{(1)}$ drives $Z_{3}^{(1)}$, and finally $Z_{3}^{(1)}$ drives $P$, see Fig. 2. Moreover, although the scalar system of $Z_{2}^{(1)}$ is affected by also other state variables of the driver, the control input may be readily designed in order to assign any chosen dynamics for $Z_{2}^{(1)}$. According to the formal development of the control design in the next Section, such a task requires a complete knowledge of the system, i.e. all the species are required to be accessible from measurements.

Finally, notice that the original system equations are interlaced in a more complex way, and the causal bindings involve couples of variables: we have that $S$ drives $C$, and $C$ drives $P$ in a simple way, but $S$ in turn is driven by the couple $(C, E)$ and $E$ is driven by the couple $(C, u)$, so there is not a direct causal binding $u \rightarrow P$ as in the case provided by the quadratic immersion.

\section{B. Design of the global regulator}

The goal is to design the control law $u$ in order to have a desired steady-state value $P_{d}$ for the product. Before to state the main theorem, we introduce the following preliminary Lemma.

Lemma 1: Assume the following constraints involving the system initial conditions hold true:

$$
\begin{gathered}
C(0)>0, \quad 0<P(0)<P_{d}<S(0)+C(0)+P(0) \\
\frac{E(0) S(0)}{C(0)}<\frac{k^{-}+v}{k^{+}}+\frac{v C(0)}{k^{+} P(0)} \\
k^{-}+v+\frac{v C(0)}{P(0)}-\frac{k^{+} E(0) S(0)}{C(0)}>\frac{v C(0)}{P(0) \ln \left(P_{d} / P(0)\right)}
\end{gathered}
$$

Then, the second order equation

$$
\alpha^{2}-\Sigma_{\alpha} \alpha+\Pi_{\alpha}=0
$$

with

$$
\Sigma_{\alpha}=2\left(k^{-}+v\right)+\frac{2 v C(0)}{P(0)}-2 \frac{k^{+} E(0) S(0)}{C(0)}
$$

and

$$
\Pi_{\alpha}=\frac{v C(0) \Sigma_{\alpha}}{2 P(0) \ln \left(P_{d} / P(0)\right)} .
$$

admits positive real roots.

Proof: The first constraints in (18) ensure meaningfulness of the other two constraints. Constraint (19) ensures that $\Sigma_{\alpha}>0$, that implies that also $\Pi_{\alpha}>0$ since $P_{d}>P(0)$. Finally, constraint (20) ensures that the discriminant $\Sigma_{\alpha}^{2}-4 \Pi_{\alpha}$ associated to (21) is positive. Hence the roots of (21) are positive real.
Notice that the first constraints in (18) includes the nonrestrictive assumption $P_{d}<S(0)+C(0)+P(0)$ that means we cannot set the desired value of the product at a value greater than the total amount of substrate, provided by $S+C+P$. Since no total substrate variations are considered, this amount coincides with the initial total amount of substrate $S(0)+$ $C(0)+P(0)$.

Theorem 2: Assume constraints (18)-(20) hold true, and design the control law $u$ as follows:

$$
u(t)=\frac{\psi(t)-\varphi_{1}(Z(t))}{\varphi_{2}(Z(t))}
$$

where $\varphi_{1}(Z), \varphi_{2}(Z)$ are defined in (16), (17), and

$$
\begin{gathered}
\psi(t)=-\frac{v \beta\left(\alpha_{1} e^{-\alpha_{1} t}+\alpha_{2} e^{-\alpha_{2} t}\right)}{k^{+}}+ \\
\frac{\left(\alpha_{1}^{2} e^{-\alpha_{1} t}+\alpha_{2}^{2} e^{-\alpha_{2} t}\right)\left(e^{-\alpha_{1} t}+e^{-\alpha_{2} t}\right)-\left(\alpha_{1} e^{-\alpha_{1} t}+\alpha_{2} e^{-\alpha_{2} t}\right)^{2}}{k^{+}\left(e^{-\alpha_{1} t}+e^{-\alpha_{2} t}\right)^{2}}
\end{gathered}
$$

with

$$
\beta=\frac{C(0)}{2 P(0)}
$$

and $\alpha_{1}, \alpha_{2}$ are the positive real roots of (21). Then, $P(t)$ converges to the desired value $P_{d}$ according to the following evolution

$$
P(t)=P(0) e^{v \beta\left(\frac{1-e^{-\alpha_{1} t}}{\alpha_{1}}+\frac{1-e^{-\alpha_{2} t}}{\alpha_{2}}\right)}
$$

Proof: The proof is given by construction: it will be shown how to design the control $u$ in order to ensure the asymptotic convergence of $P$ to the desired value. To this end, according to (13), we aim to set the control law in order to ensure the following shape for $Z_{3}^{(1)}$ :

$$
Z_{3}^{(1)}(t)=\beta\left(e^{-\alpha_{1} t}+e^{-\alpha_{2} t}\right), \quad \alpha_{1}, \alpha_{2}, \beta>0
$$

with parameters $\alpha_{1}, \alpha_{2}$ and $\beta$ to be assigned. Indeed, if eq.(28) holds true, by substituting it in (13) we have:

$$
\dot{P}(t)=v \beta\left(e^{-\alpha_{1} t}+e^{-\alpha_{2} t}\right) P(t)
$$

whose explicit solution provides, after computations, eq.(27). Thus, if $P_{d}$ is the desired steady-state value for $P(t)$, the following constraint for the control parameters is given:

$$
\begin{aligned}
& \lim _{t \mapsto+\infty} P(t)=P(0) e^{v \beta\left(\frac{1}{\alpha_{1}}+\frac{1}{\alpha_{2}}\right)}=P_{d} \\
& \Longrightarrow \quad v \beta\left(\frac{1}{\alpha_{1}}+\frac{1}{\alpha_{2}}\right)=\ln \left(\frac{P_{d}}{P(0)}\right)>0
\end{aligned}
$$

Inequality $\ln \left(P_{d} / P(0)\right)>0$ is ensured by the fact that $P_{d}>$ $P(0)$, according to the constraint (18). As a matter of fact, the control law can force the product concentration to increase only.

A second constraint comes from the initial value of $Z_{3}^{(1)}$ since, according to (28):

$$
Z_{3}^{(1)}(0)=\frac{C(0)}{P(0)}=2 \beta \quad \Longrightarrow \quad \beta \text { as in (26) }
$$


In order to have the explicit solution for $Z_{3}^{(1)}(t)$ as the one defined by (28), we substitute

$$
\dot{Z}_{3}^{(1)}(t)=-\beta\left(\alpha_{1} e^{-\alpha_{1} t}+\alpha_{2} e^{-\alpha_{2} t}\right)
$$

in (14), that becomes:

$$
\begin{aligned}
k^{+} Z_{3}^{(1)}(t) Z_{2}^{(1)}(t)-\left(k^{-}+v\right) & Z_{3}^{(1)}(t)-v\left(Z_{3}^{(1)}\right)^{2}(t) \\
& =-\beta\left(\alpha_{1} e^{-\alpha_{1} t}+\alpha_{2} e^{-\alpha_{2} t}\right)
\end{aligned}
$$

which entails, after computations, the following explicit expression for $Z_{2}^{(1)}$

$$
\begin{aligned}
Z_{2}^{(1)}(t)=\frac{k^{-}+v}{k^{+}}+ & \frac{v \beta}{k^{+}}\left(e^{-\alpha_{1} t}+e^{-\alpha_{2} t}\right) \\
& -\frac{1}{k^{+}} \frac{\alpha_{1} e^{-\alpha_{1} t}+\alpha_{2} e^{-\alpha_{2} t}}{e^{-\alpha_{1} t}+e^{-\alpha_{2} t}}
\end{aligned}
$$

with a third constraint given by the initial condition:

$$
Z_{2}^{(1)}(0)=\frac{E(0) S(0)}{C(0)}=\frac{k^{-}+v}{k^{+}}+\frac{2 v \beta}{k^{+}}-\frac{\alpha_{1}+\alpha_{2}}{2 k^{+}}
$$

Besides $\beta$, straightforwardly given by (31), constraints (30) and (34) allow to univocally set parameters $\alpha_{1}, \alpha_{2}$. Notice that these two constraints readily provide the solutions in terms of sum and product of $\alpha_{1}$ and $\alpha_{2}$. Indeed, by denoting

$$
\Sigma_{\alpha}=\alpha_{1}+\alpha_{2} \quad \Pi_{\alpha}=\alpha_{1} \alpha_{2}
$$

constraints (30) and (34) allow to write $\Sigma_{\alpha}, \Pi_{\alpha}$ as in (22) and (23), and Lemma 1 guarantees that eq.(21) provides real and positive solutions.

Finally, the control law $u$ is derived from (15) by substituting the derivative $\dot{Z}_{2}^{(1)}$ with its analytic expression coming from the derivative of (33). After computations, eq.(24) with (25) is obtained.

Remark 3: Notice that inequalities (19), (20) could be replaced by the following

$$
K_{M}>\frac{E(0) S(0)}{C(0)}, \quad \quad P_{d}>e P(0)
$$

where $K_{M}=\left(k^{-}+v\right) / k^{+}$is the Michaelis Menten coefficient. However, the inequalities in (36), though easier to check and to deal with, are more conservative, since they are sufficient conditions for (19), (20).

Remark 4: Regards to constraints in (18), they should be here considered as non restrictive constraints. Differently from the usual setting for a basic enzymatic reaction scheme (see, among the others, [22]) the chemical reaction network here investigated is not closed. Therefore nontrivial initial values of the complex and of the product are biologically meaningful. From a mathematical viewpoint, such constraints derive from the quadratic immersion, defined on all the original system domain, with the exception of a zero measure set, where the driver state is not defined. This set, in our problem, corresponds to $P=0$, a zero concentration of the product. This may not be actually a serious limitation, in that we have excluded just a case of an exactly zero quantity of initial product. Moreover, such a limitation could be removed by expressing the original system in a new set of coordinates
TABLE I

MODEL PARAMETERS, ARBITRARY UNITS.

\begin{tabular}{|c|c|}
\hline Parameter & Value \\
\hline$k^{+}$ & 1 \\
\hline$k^{-}$ & 15 \\
\hline$v$ & 5 \\
\hline
\end{tabular}

TABLE II

INITIAL CONDITIONS, ARBITRARY UNITS.

\begin{tabular}{|c|c|}
\hline Initial condition & Value \\
\hline$S(0)$ & 10 \\
\hline$E(0)$ & 0 \\
\hline$C(0)$ & 1 \\
\hline$P(0)$ & 1 \\
\hline
\end{tabular}

where the zero concentration is moved to some non zero value in the new coordinates. However, this complicates the system equations, and leads actually to another problem, whose solution has no more the simplicity of the method here proposed.

\section{Simulations}

Simulations have been carried out in order to test the theoretical results, with model parameters and initial conditions set as in Table I and Table II, respectively. The desired value for the product concentration is $P_{d}=7$.

Simulations are drawn in Fig.3, where the four chemical players are reported. Fig.4 reports the production rate of the enzyme (i.e. the control law $u$ ). Notice that it becomes negative for a period, meaning that the enzyme is required to be spilled out of the reactor, according to the designed control law.

Fig. 5 reports the same evolution of Fig. 3 (without the complex, and on a larger time scale), together with the controlfree evolution (in dashed lines). It clearly appears the different behavior, with the product in free evolution accumulating at a higher (than $P_{d}=7$ ) value, corresponding to the initial total amount of substrate, complex and product $S(0)+C(0)+$ $P(0)=12$.

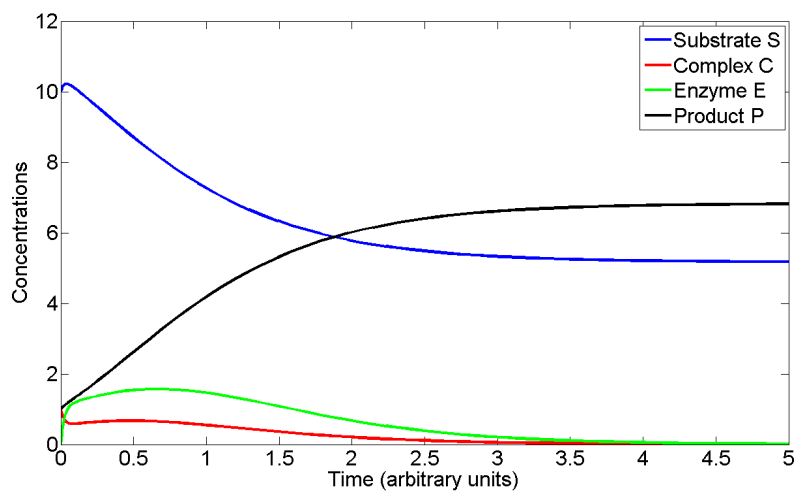

Fig. 3. Evolutions of the four chemical players' concentrations. 


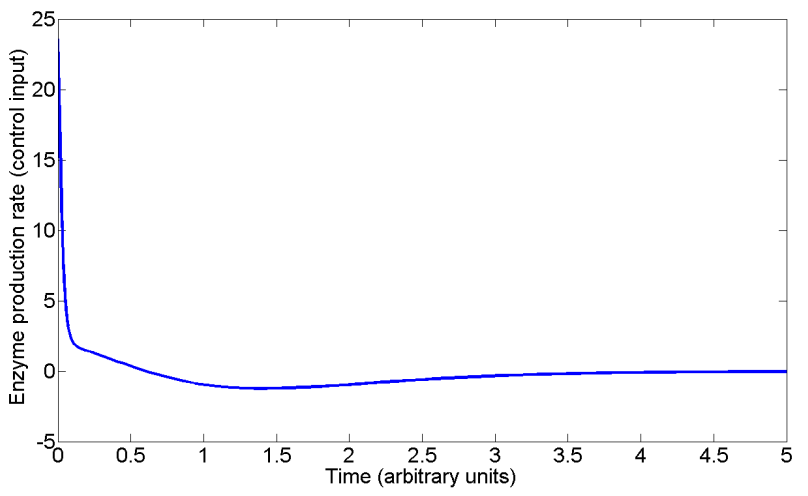

Fig. 4. Control law evolution

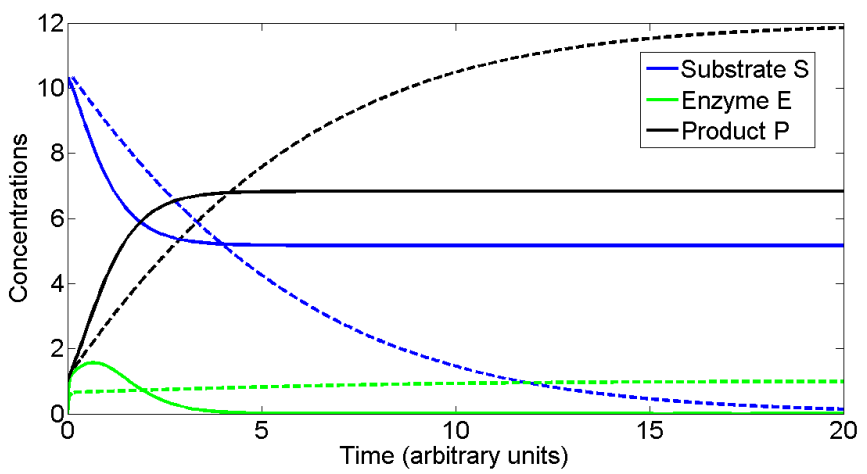

Fig. 5. Comparison between evolution with (continuous line) and without (dashed line) control.

\section{CONCLUDING REMARK.}

The regulation problem for a general set of enzymatic chemical reactions has been here considered and a closed-loop solution, with exponential performance, has been proposed. Actually, the synthesis of the control law requires the knowledge of the initial conditions, so that it can be considered a feedback/feedforward law. The considered type of system is usually found in biochemistry, nonetheless, the same set of equations describes any chemical process where a production is activated in a substrate by some catalyzer, and thus it is amenable for modeling many types of industrial reactors as well. By using the quadratic immersion we have been able to perform a decoupling of the original system, and this has been the key for finding a solution to the regulation problem. The present paper represents a first step where we have mainly shown the capability of the method based on the quadratic immersion for solving nonlinear problems. For the specific problem of enzymatic reactions control, further work is needed in order to improve the performance, for instance by making tunable the convergence rate of the exponential shape of the controlled variable.

\section{ACKNOWLEDGEMENTS}

This work is supported by the MIUR grant SysBioNet Italian Roadmap for ESFRI Research Infrastructures, SYSBIO
Centre of Systems Biology, Milan and Rome, Italy.

\section{REFERENCES}

[1] M. Bardi, I. Capuzzo-Dolcetta, Optimal Control and Viscosity Solutions to Hamilton-Jacobi-Bellman Equations, Boston, MA: Birkhauser, 1997.

[2] R. W. Beard, G. N. Saridis, and J. T.Wen, Galerkin approximations of the generalized Hamilton-Jacobi-Bellman equation, Automatica, 33(12) 21592177, 1997.

[3] Briat, C., Gupta, A., Khammash, M. (2016). Antithetic integral feedback ensures robust perfect adaptation in noisy biomolecular networks. Cell systems, 2(1), 15-26.

[4] D.E. Cameron, C.J. Bashor, and J.J. Collins, A brief history of synthetic biology, Nat. Rev. Microbiol., 12, pp. 381-390, 2014.

[5] F. Carravetta, Some Results on the Problem of Global Exact Bilinearization for Nonlinear Delay Systems, 51st IEEE Conference on Decision and Control (CDC '12) Maui, Hawaii, USA, December 10-13. 2012.

[6] F. Carravetta, Almost-Global Exponential State-Feedback Stabilization of an Underactuated Rigid-Body in 3D, Proc. of the 53rd IEEE Conference on Decision and Control, (CDC 2014) Los Angeles, California, USA, December 15-17, 2014.

[7] F. Carravetta, Global Exact Quadratization of Continuous-Time Nonlinear Control Systems, SIAM J. Control Optim., 53(1) 235-261, 2015.

[8] R. Chang, Physical chemistry for the chemical and biological sciences, University Science Books, 2000.

[9] G. Cooper, The Cell: A molecular approach, USA: Sinauer Associates, 2000.

[10] C. Cosentino, R. Ambrosino, M. Ariola, M. Bilotta, A. Pironti, and F. Amato, On the realization of an embedded subtractor module for the control of chemical reaction networks, IEEE Trans. Automatic Control, 61(11), pp. 3638-3643, 2016.

[11] D. Del Vecchio, Modularity, context-dependence, and insulation in engineering biological circuits, Trends in Biotechnology, 33(2), 111119, 2015.

[12] D. Del Vecchio, A.J. Dy, Y. Qian, Control theory meets synthetic biology, J. R. Soc. Interface, 13: 20160380. http://dx.doi.org/10.1098/rsif.2016.0380

[13] D. Del Vecchio, A. J. Ninfa, and E. D. Sontag, Modular cell biology: retroactivity and insulation, Molecular systems biology, 4.1, 161, 2008.

[14] D. Del Vecchio, and E. D. Sontag, Synthetic biology: A systems engineering perspective, "Control Theory and Systems Biology", P.A. Iglesias \& B.P. Ingalls Eds., The MIT Press, 101-124, 2009.

[15] M.J. Dunlop, J.D. Keasling, and A. Mukhopadhyay, A model for improving microbial biofuel production using a synthetic feedback loop, Syst. Synth. Biol., 4, pp. 95-104, 2010.

[16] M. Fliess, I. Kupka, A finiteness Criterion for Nonlinear Input-Output Differential Systems, SIAM J. Control Optim. 21, 721 - 728, 1983.

[17] Isidori, A.: Nonlinear Control Systems, Springer, London, 1995

[18] Z.P. Jiang, A. Teel, L. Praly, Small-gain theorem for ISS systems and applications, Mathematics of Control, Signals, and Systems 7 95-120, 1994.

[19] P. Jouan, Immersion of Nonlinear Systems into Linear Systems Modulo Output Injection, SIAM J. Control Optim. 41, 1756 - 1778, 2003.

[20] O. Kirk, T.V. Borchert, C.C. Fuglsang, Industrial enzyme applications, Curr. Opin. Biotechnol., 13, 345-351, 2002.

[21] M. Sassano, A. Astolfi, Dynamic Approximate Solutions of the HJ Inequality and of the HJB Equation for Input-Affine Nonlinear Systems, IEEE Trans. on Aut. Cont., 57(10) 2490-2503, 2012.

[22] Segel, L.: On the validity of the steady state assumption of enzyme kinetics. Bull. Math. Biol. 50, 579593 (1988)

[23] E.D. Sontag, Y. Wang, On Characterization of Input-to-State Stability, Syst. \& Cont. Letters, 24 351-359, 1995.

[24] J.A. Stapleton, K. Endo, Y. Fujita, K. Hayashi, M. Takinoue, H. Saito, and $\mathrm{T}$. Inoue, Feedback control of protein expression in mammalian cells by tunable synthetic translational inhibition, ACS Synth. Biol., 1, pp. 83-88, 2012.

[25] N.G. Van Kampen, Stochastic processes in physics and chemistry, 3rd ed. Amsterdam, The Netherlands: North Holland, 1992. 